\title{
Relationship Between Jump Landing Kinematics and Peak ACL Force During a Jump in Downhill Skiing: A Simulation Study
}

\author{
D. Heinrich \\ University of Innsbruck
}

Antonie J. van den Bogert

Cleveland State University, a.vandenbogert@csuohio.edu

W. Nachbauer

University of Innsbruck

Follow this and additional works at: https://engagedscholarship.csuohio.edu/enme_facpub

Part of the Biomechanical Engineering Commons

How does access to this work benefit you? Let us know!

\section{Publisher's Statement}

This is the accepted version of the following article: Heinrich, D., van den Bogert, A. J., and Nachbauer, W., 2014, "Relationship between Jump Landing Kinematics and Peak ACL Force during a Jump in Downhill Skiing: A Simulation Study," Scandinavian Journal of Medicine \& Science in Sports, 24(3) pp. e180-e187, which has been published in final form at http://onlinelibrary.wiley.com/doi/10.1111/sms.12120/abstract

\section{Original Citation}

Heinrich, D., van den Bogert, A. J., and Nachbauer, W., 2014, "Relationship between Jump Landing Kinematics and Peak ACL Force during a Jump in Downhill Skiing: A Simulation Study," Scandinavian Journal of Medicine \& Science in Sports, 24(3) pp. e180-e187.

This Article is brought to you for free and open access by the Mechanical Engineering Department at EngagedScholarship@CSU. It has been accepted for inclusion in Mechanical Engineering Faculty Publications by an authorized administrator of EngagedScholarship@CSU. For more information, please contact library.es@csuohio.edu. 


\title{
Relationship between jump landing kinematics and peak ACL force during a jump in downhill skiing: A simulation study
}

\author{
D. Heinrich ${ }^{1,2}$, A. J. van den Bogert ${ }^{3,4}$, W. Nachbauer ${ }^{1}$ \\ ${ }^{1}$ Department of Sport Science, University of Innsbruck, Innsbruck, Austria, ${ }^{2}$ Centre of Technology of Ski and Alpine Sports, Innsbruck, \\ Austria, ${ }^{3}$ Orchard Kinetics LLC, Cleveland, Ohio, USA, ${ }^{4}$ Department of Mechanical Engineering, Cleveland State University, \\ Cleveland, Ohio, USA \\ Corresponding author: Dieter Heinrich, Department of Sport Science, University of Innsbruck, Innsbruck, Austria. Tel.: +43 512 507 \\ 4467, Fax: +43 512507 2656, E-mail: dieter.heinrich@uibk.ac.at
}

\begin{abstract}
Recent data highlight that competitive skiers face a high risk of injuries especially during off-balance jump landing maneuvers in downhill skiing. The purpose of the present study was to develop a musculo-skeletal modeling and simulation approach to investigate the cause-andeffect relationship between a perturbed landing position, i.e., joint angles and trunk orientation, and the peak force in the anterior cruciate ligament (ACL) during jump landing. A two-dimensional musculo-skeletal model was developed and a baseline simulation was obtained reproducing measurement data of a reference landing movement. Based on the baseline simulation, a series of perturbed landing simulations $(n=1000)$ was generated.
\end{abstract}

Multiple linear regression was performed to determine a relationship between peak ACL force and the perturbed landing posture. Increased backward lean, hip flexion, knee extension, and ankle dorsiflexion as well as an asymmetric position were related to higher peak ACL forces during jump landing. The orientation of the trunk of the skier was identified as the most important predictor accounting for $60 \%$ of the variance of the peak ACL force in the simulations. Teaching of tactical decisions and the inclusion of exercise regimens in ACL injury prevention programs to improve trunk control during landing motions in downhill skiing was concluded.
In the last few years, injury data reported through the International Ski Federation Injury Surveillance System highlight that competitive skiers face a high injury risk (Flørenes et al. 2009, 2012). During the winter seasons from 2006 to 2008, about every third athlete suffered a time loss injury and about every ninth athlete suffered a severe injury per season ( $>28$ days of absence). Severe injuries typically involve the knee and knee ligaments and injuries of the anterior cruciate ligament (ACL) are the most frequent specific diagnosis (Flørenes et al., 2009). Among the different skiing disciplines, the highest injury rate has been reported in downhill skiing (Flørenes et al., 2009) and landing from a jump has been identified as the dominant ACL injury mechanism in downhill skiing (Bere et al., 2011a).

Recent studies focusing on video analysis of injury cases have provided considerable insight into possible mechanisms of ACL injuries (Bere et al., 2011a) and the events leading to the injury situation during the landing movement (Bere et al., 2011b). In the study of Bere et al. (2011b), skiers' mistakes such as a poor jumping technique and incorrect tactical decisions have been determined as key factors contributing to injury. These mistakes have been observed to result in a backward off-balance situation of the skier during the flight and in landing on the tails of the skis with nearly extended knees. However, there is a lack of knowledge, how these characteristics are related to the loading of the knee and knee ligaments and whether the kinematics of the skier prior to jump landing might be used to predict the peak ACL force during the subsequent landing. In addition, knowledge of the most important predictor(s) might be included in the design of prevention programs and the teaching of tactical decisions with the aim to reduce the number of ACL injuries during jump landing in downhill skiing.

Because of ethical reasons, in vivo studies, which mimic possible injury situations, are not feasible. However, musculo-skeletal modeling and simulation offer the possibility to study injury situations in a computer environment and estimate ACL forces and strains (Krosshaug et al., 2005). In addition, sophisticated musculo-skeletal models allow the investigation of cause-and-effect relations such as between neuromuscular control and knee joint loading using a series of Monte Carlo simulations (McLean et al., 2004, 2008). McLean et al. (2004) used as series of Monte Carlo simulations to study random perturbations in initial trunk and joint kinematics on knee joint loading during a side-step cutting task. In a subsequent study, McLean et al. (2008) 
analyzed modifications in initial hip and knee flexion, hip internal rotation, and hip internal rotation velocity on knee joint loading in a series of side-step cutting simulations. In the simulation study of Gerritsen et al. (1996), a possible injury situation was investigated during jump landing in downhill skiing. A single backward offbalance landing movement in combination with an active recovery movement was simulated and the peak force in the ACL was predicted. However, up to now, there is a lack of simulation studies focusing on a systematic analysis of the relationship between the kinematics of the skier prior to ground contact and the subsequent peak ACL force during jump landing in downhill skiing.

The purpose of the present study was to develop a musculo-skeletal modeling and simulation approach to investigate the effect of a perturbed landing position, i.e., joint angles and trunk orientation, on peak ACL force during the subsequent landing movement.

\section{Methods}

\section{Musculo-skeletal model}

A planar, musculo-skeletal model of an alpine skier with two skis was developed. The skier model consisted of seven rigid segments, one segment representing the head, arms and torso, and three segments for each lower extremity: thigh, shank, and one segment for the foot and the ski boot. The restraining component of the ski boot was represented as passive joint moment acting at the ankle joint (Gerritsen et al., 1996). The model of each ski consisted of a chain of nine rigid segments connected by revolute joints. Bending stiffness and damping properties of the skis were integrated into the model using linear spring-damper elements attached to the revolute joints. Parameters of the spring-damper elements were derived from bending experiments and ski vibration tests (Bruck et al., 2003) of a downhill ski with a nominal length of $2.11 \mathrm{~m}$. Multibody dynamics was derived based on Kane's method using Autolev 4.1 (Symbolic Dynamics Inc., Sunnyvale, California, USA). In total, the skier-skis system had 25 kinematic degrees of freedom and the equations of motion were formulated as:

$$
\boldsymbol{M}(\boldsymbol{q}) \ddot{\boldsymbol{q}}+\boldsymbol{B}(\boldsymbol{q}, \dot{\boldsymbol{q}})+\boldsymbol{R}(\boldsymbol{q}) F_{M}=0
$$

where $\boldsymbol{q}, \dot{\boldsymbol{q}}$, and $\ddot{\boldsymbol{q}}$ are the generalized coordinates, velocities, and accelerations; $\boldsymbol{M ( q )}$ is the system mass matrix; $\boldsymbol{B}(\boldsymbol{q}, \dot{\boldsymbol{q}})$ is a vector of generalized forces due to gravity, external forces and moments, coriolis and centrifugal forces and moments, and passive joint moments; $\boldsymbol{R}(\boldsymbol{q})$ is a matrix of muscle moment arms; $F_{M}$ is a vector of muscle forces and $\boldsymbol{R}(\boldsymbol{q}) \boldsymbol{F}_{\boldsymbol{M}}$ is a vector of net joint moments.

The motion of the skier was actuated by 16 muscles, eight for each lower extremity: iliopsoas (Ili), glutei (Glu), hamstrings (Ham), rectus femoris (RF), vasti (Vas), gastrocnemius (Gas), soleus (Sol), and tibialis anterior (TA). Each muscle was modeled as three-element Hill-type model. Contraction dynamics incorporating the force-length-velocity properties of the muscle was modeled as function of muscle fiber length, $L_{C E}$, fiber lengthening velocity, $\dot{L}_{C E}$, activation, $a$, and muscle length, $L_{M}(q)$ (McLean et al., 2003),

$$
\boldsymbol{g}\left(L_{\boldsymbol{C E}}, \dot{L}_{\boldsymbol{C E}}, a, L_{M}(\boldsymbol{q})\right)=0
$$

Activation dynamics was modeled as a first-order process (He et al., 1991):

$$
\boldsymbol{h}(\boldsymbol{a}, \dot{\boldsymbol{a}}, \boldsymbol{u})=0
$$

where $\boldsymbol{u}$ denotes the neural excitation of the muscles. A linear relationship was assumed between joint angles and muscle-tendon length (McLean et al., 2003) and muscle parameters were taken from Gerritsen et al. (1996). In total, the musculo-skeletal model of the skier and the two skis had 82 state variables $\boldsymbol{x}=\left[\boldsymbol{q}, \dot{\boldsymbol{q}}, \boldsymbol{a}, L_{C E}\right]^{T}$ and 16 control variables $\boldsymbol{u}$. The dynamics of the skier-skis model was given by eqns. [1], [2], and [3].

\section{Contact models}

Contact between skis and snow was modeled by two types of forces applied at the mass center of each ski segment: a penetration force, $F_{p}$, acting normal to the snow surface and a friction force, $F_{f}$, acting parallel to the snow surface. The penetration force was given by the following equation:

$$
F_{p}=k_{p} A\left(\frac{1}{2} d+\frac{1}{2} \sqrt{d^{2}+d_{0}^{2}}\right)\left(1+b_{d} \dot{d}\right)
$$

where $d$ and $\dot{d}$ are the penetration depth and velocity of the segment's center of mass, $A$ is the area of the rectangularly approximated base of the segment, and $k_{p}$ and $b_{p}$ are ski-snow contact parameters. The stiffness parameter $k_{p}$ was set to $6 \times$ $10^{5} \mathrm{~N} / \mathrm{m}^{3}$ to get a maximum penetration of about $0.05 \mathrm{~m}$ at the ski below the area of the ski boot. In reality, the penetration below the area of the ski boot corresponds to the deformation of the heel pad, the liner of the ski boot, the ski bindings, and the snow. The damping parameter $b_{p}$ was set to $0.75 \mathrm{~s} / \mathrm{m}$. The parameter $d_{0}$ represents a smooth transition region of the contact model and was set to $0.01 \mathrm{~m}$. The friction force $F_{f}$ was approximated by Coulomb friction with a friction coefficient of $\mu=0.1$ (Kaps et al., 1996):

$$
F_{f}=-\mu F_{p} \frac{v}{\sqrt{v^{2}+v_{0}^{2}}}
$$

where $v$ denotes the sliding velocity parallel to the snow surface. The smoothing parameter $v_{0}$ was set to $1 \mathrm{~m} / \mathrm{s}$. Air drag was applied on each segment of the skier based on the regression model of Barelle et al. (2004), which describes the relationship between total drag force and the skier's body size and posture.

\section{Baseline simulation of landing movement}

A baseline simulation was obtained in which muscle stimulation patterns of the model were optimized to track a reference landing movement in competitive downhill skiing. In particular, the following optimal control problem was solved, minimizing the cost function:

$$
J=J_{1}+J_{2}+J_{3}
$$

with

$$
\begin{gathered}
J_{1}=\frac{1}{N_{d} T} \int_{0}^{T} \sum_{i=1}^{N_{d}}\left(\frac{q_{i}(t)-q_{i, d a t a}(t)}{\sigma_{i}}\right)^{2} d t \\
J_{2}=\frac{w_{\text {mus }}}{N_{m} T} \int_{0}^{T} \sum_{i=1}^{N_{m}} u_{i}(t)^{2} d t
\end{gathered}
$$

and

$$
J_{3}=\frac{w_{s k}}{2 N_{s k} T} \int_{0}^{T} \sum_{i=N_{d}+1}^{N_{d}+N_{s k}}\left(q_{i}(t)^{2}+\dot{q}_{i}(t)^{2}\right) d t
$$

subjected to constraints because of the dynamics of the skier-skis model and upper and lower bounds on the controls $0 \leq \boldsymbol{u} \leq 1 . N_{d}$, 
$N_{m u s}$, and $N_{s k}$ denote the number of degrees of freedom of the skier model, the number of muscles, and the number of degrees of freedom of both skis, respectively. Time duration is represented by $T$. The first term, $J_{1}$, represents the mean deviation of the simulation results $q_{i}(t)$ with respect to experimental kinematic data $q_{i, \text { data }}(t)$ scaled by a factor $\sigma_{i}\left(1 \leq i \leq N_{d}\right)$. Experimental data were taken from a previous study (Nachbauer et al., 1996) where a downhill skier was captured with two high-speed cameras at $180 \mathrm{~Hz}$ performing a jump landing movement that lasted for $1 \mathrm{~s}$. Anthropometric measurements of the downhill skier and geometric modeling were used to derive segment length, segment mass, and inertia properties of the skier model. The second term, $J_{2}$, weighted with $w_{\text {mus }}$, was used to resolve muscle redundancy as in Spägele et al. (1999). The third term, $J_{3}$, weighted with $w_{s k}$, was used to avoid excessive ski bending and vibration especially during the aerial phase.

\section{Solution method}

The optimal control problem was transformed into a constrained nonlinear programming problem (NLP) using direct collocation (Betts, 2010) and the implicit midpoint formula for discretization of the system dynamics (van den Bogert et al., 2011). The NLP was solved using IPOPT (Wächter \& Biegler, 2006), an interior point optimization solver, and a mesh refinement strategy. The weight factor $w_{\text {mus }}=10$ was found to give a reasonable weighting between $J_{1}$ and $J_{2} . J_{3}$ was relatively small and was weighted by $w_{s k}=0.01$.

\section{Knee joint loading}

Sagittal knee joint loading was calculated similar to the study of McLean et al. (2008). In addition, the slope of the tibial plateau was included in the present calculations as follows. The anteriorposterior resultant knee reaction force with respect to the tibial plateau was calculated from the simulated movement, gravity, and the contact forces using a standard inverse dynamics analysis (Winter, 2009). A tibial slope angle of $9^{\circ}$ was assumed (Matsuda et al., 1999). The relative contributions of the quadriceps and hamstring muscle forces were subtracted from the resultant knee reaction force to obtain an estimate of the anterior drawer force. Based on the anterior drawer force, cruciate ligament forces (ACL and posterior cruciate ligament, respectively) were calculated by assuming that only one cruciate at a time is loaded. In the calculations, muscle and ligament orientations were defined as a function of knee flexion angle using the data of Herzog \& Read (1993).

\section{Perturbed landing simulations}

Based on the baseline simulation a series of Monte Carlo simulations $(n=1000)$ was generated to investigate the effect of a perturbed landing position prior to ground contact on peak ACL force during the subsequent landing movement. During the subsequent landing movement, it was assumed that the skier attempts an active recovery movement with the aim to regain balance. Specifically, in each simulation of the Monte Carlo series, the kinematic state of the skier obtained by the baseline simulation $0.4 \mathrm{~m}$ above the snow surface was perturbed by adding random numbers to the trunk orientation and to the joint angles of the left and right lower extremity. The random numbers were generated assuming a Gaussian distribution with zero mean and standard deviations derived from the study of Barone et al. (1999) (hip: $9.4^{\circ}$; knee: $8.7^{\circ}$; ankle: $5.2^{\circ}$; trunk orientation: $9.2^{\circ}$ ). The distance of $0.4 \mathrm{~m}$ was chosen to avoid that the ski tails penetrate the snow in the perturbed initial position. With the initial posture of the skier constrained to the perturbed values an optimal control problem similar to the baseline simulation was solved to simulate the recov- ery movement. In contrast to the baseline simulation, muscle stimulation patterns were optimized to track the human landing data only at the final time, where the skier was observed to be in a well-balanced downhill position. Correspondingly, the term $J_{1}$ was replaced by the deviation of the measurement and simulation data at the final time $t=1$ in the cost function. Additionally, the weight factor $w_{\text {mus }}$ was reduced to 1 to increase the weight with respect to the tracking of the balanced end position. Regaining a balanced position represents the behavior that would be expected from a real skier who finds him/herself in a perturbed position prior to ground contact.

\section{Statistical analyses}

Multiple linear regression was performed to investigate the relationship between the peak force in the ACL in the left/right lower extremity during the landing movement as dependent variable and perturbed kinematic state variables of the posture of the skier prior to ground contact as independent variables. The perturbed kinematic state variables were the orientation of the trunk segment, the joint angles at the hip, knee, and ankle of the lower extremity corresponding to the peak force in the ACL as well as the difference in the joint angles at the hip, knee, and ankle of both lower extremities. Instead of the joint angles of the second lower extremity, these differences were included in the analysis to account for asymmetries in the posture of the skier. Bivariate correlations were also performed to further examine the relationship between the kinematic posture variables and peak force in the ACL. Finally, squared semipartial correlations were evaluated to determine the amount of variance in the dependent variable attributed uniquely to each independent variable (Trabachnick \& Fidell, 2005).

\section{Results}

The baseline simulation could be successfully solved (Fig. 1, an animation is provided online as supporting information) and repeated runs with different random initial guesses resulted in identical solutions. Joint angles and trunk orientation of the skier obtained by the baseline simulation were in good agreement with the corresponding measurement data, with differences similar to the noise in the measurements (Fig. 2). Root mean square (RMS) differences between measured and optimized joint angles ranged from $2.9^{\circ}$ to $6.7^{\circ}$; the RMS difference of the trunk orientation was $3.7^{\circ}$.

In the baseline simulation, primarily, the right knee of the skier was loaded. In the right knee, the ACL was loaded until $60 \mathrm{~ms}$ after ground contact when the knee flexed from $36.3^{\circ}$ to $66.0^{\circ}$. Ground contact was defined at time $t=0.46 \mathrm{~s}$ when the ground reaction force at the ski segment below the ski boot exceeded $10 \mathrm{~N}$. In the right knee, peak ACL force reached $695 \mathrm{~N}$ after $33 \mathrm{~ms}$ of ground contact; in the left knee, peak ACL force amounted to $165 \mathrm{~N}$ (Fig. 3). In the Monte Carlo simulations $(n=1000)$, substantially higher peak ACL forces were observed compared with the baseline simulation (Fig. 4) reaching values up to $2017 \mathrm{~N}$.

The multiple regression analysis showed that the posture variables of the skier prior to ground contact significantly predicted the peak force in the ACL during the subsequent landing movement $[F(7,951)=956.34$, 

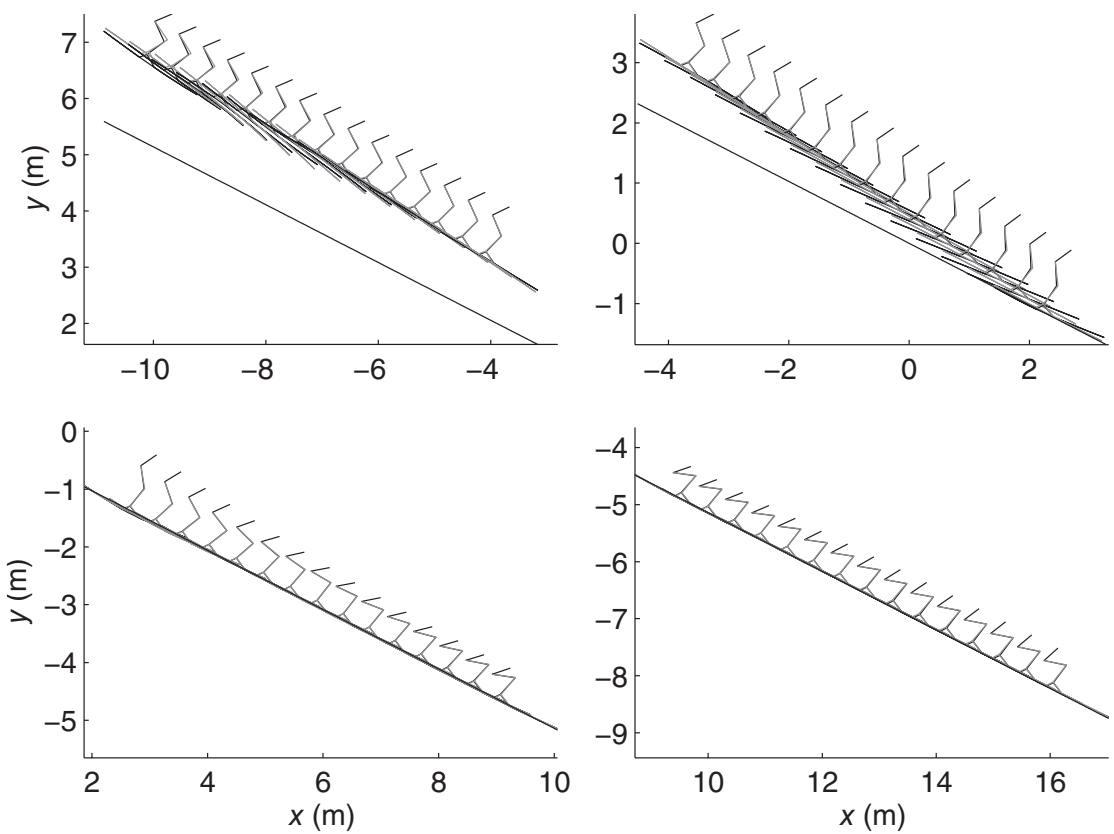

Fig. 1. Stick figure diagram of the baseline landing simulation $(\Delta t=1 / 180)$ showing every third frame.
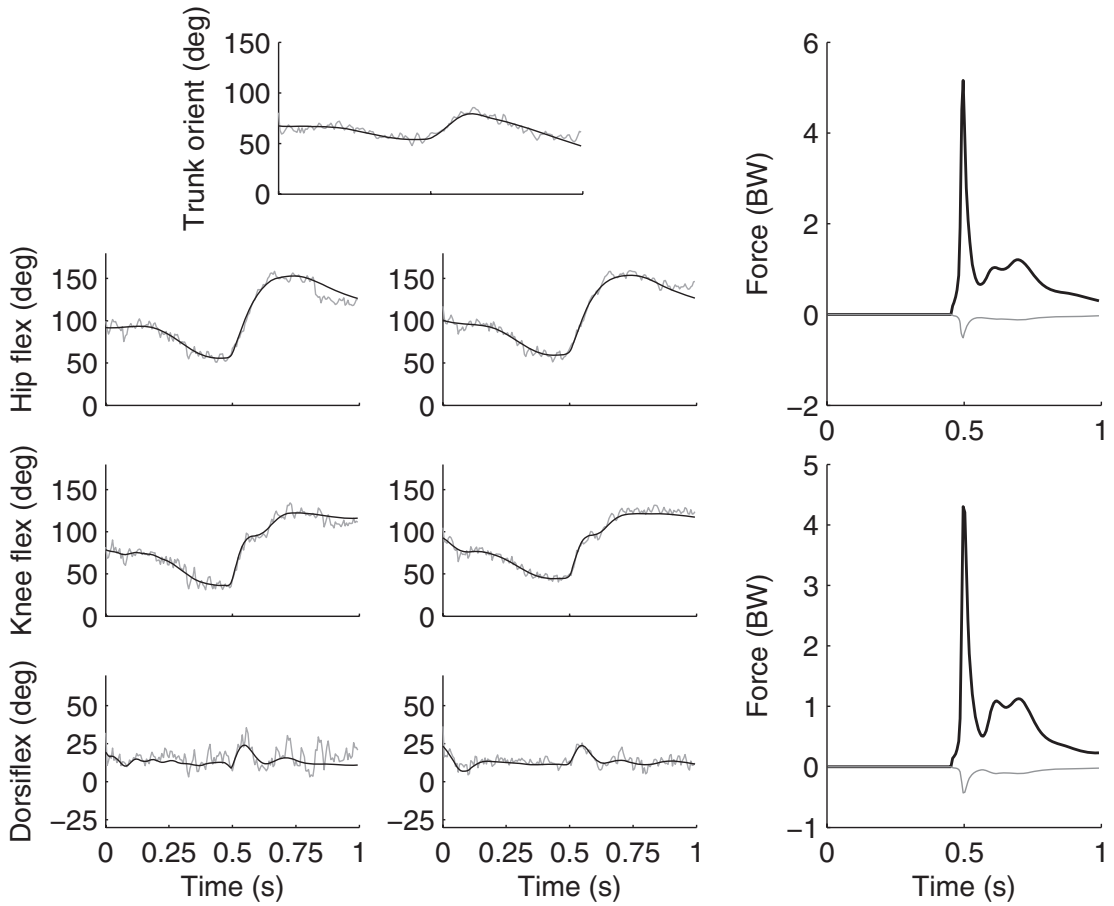

Fig. 2. Comparison of optimized joint angles (black) and measured joint angles (gray) in the right and left lower extremity (first and second column). Ground reaction forces normal to the snow surface (black) and parallel to the snow surface (gray) obtained in the optimization are shown in the third column (right leg above).

$\left.P<0.001, R^{2}=0.88\right]$. In the regression model, the orientation of the trunk, the joint angles at the hip, knee and ankle of the loaded lower extremity, and the difference in hip and knee flexion were included (Table 1) accounting for $88 \%$ of the variance of the peak force in the ACL. Although the bivariate correlations (Table 2) showed significant relations between each independent variable and the dependent variable, the difference in ankle dorsiflexion did not contribute significantly to the regression.

The individual regression coefficients revealed that increased backward lean, hip flexion, knee extension, and ankle dorsiflexion were related to higher peak ACL forces during the subsequent landing movement. Differences in hip and knee flexion angles, which account for 


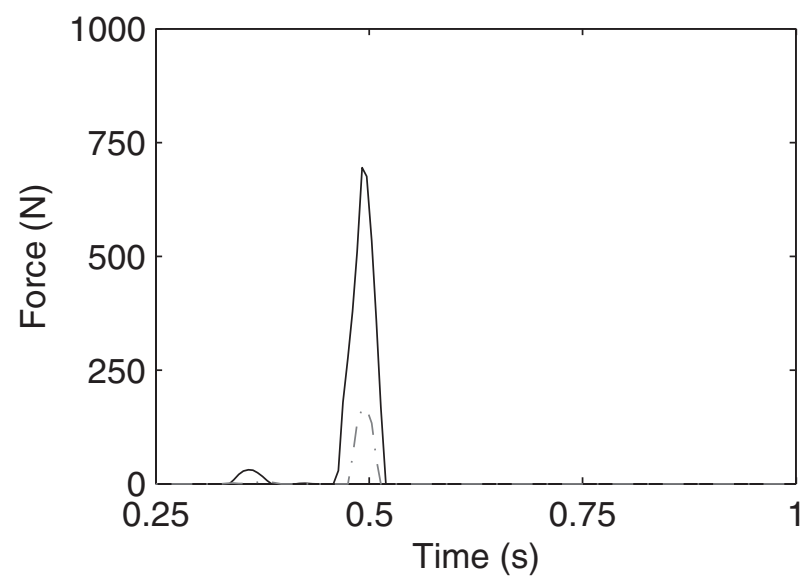

Fig. 3. ACL force in the right (solid) and left knee (dash-dotted) in the baseline landing simulation.

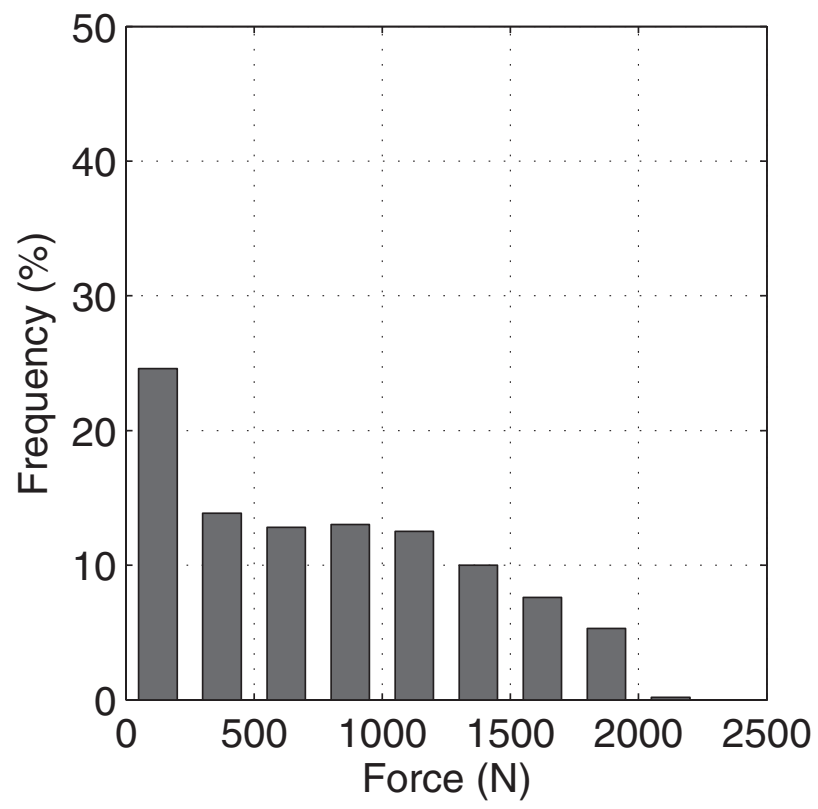

Fig. 4. Distribution of peak ACL force obtained by the series of Monte Carlo simulations $(n=1000)$.

an asymmetric position, were also related to increased peak ACL forces (Table 2).

The orientation of the trunk of the skier was identified as the most important predictor in the regression model. As indicated by the squared semipartial correlations, $60 \%$ of the variance of the peak force in the ACL could be uniquely attributed to the orientation of the trunk segment prior to ground contact (Table 3).

\section{Discussion}

The purpose of the present study was to develop a musculo-skeletal modeling and simulation approach to investigate the effect of a perturbed landing position, i.e., joint angles and trunk orientation, on peak ACL force during the subsequent landing movement.
Table 1. Results of the multiple linear regression analysis showing the unstandardized coefficients $B$ and standardized coefficients $\beta$ of the predictor variables. In the multiple linear regression analysis, the peak force in the ACL in the left/right lower extremity during the landing movement was chosen as dependent variable and perturbed kinematic state variables of the posture of the skier prior to ground contact as the predictor variables. The perturbed kinematic state variables were the orientation of the trunk segment, the joint angles at the hip, knee, and ankle of the lower extremity corresponding to the peak force in the ACL as well as the difference in the joint angles at the hip, knee, and ankle of both lower extremities

\begin{tabular}{lrrr}
\hline Variable & \multicolumn{1}{l}{$B$} & \multicolumn{1}{l}{$\beta$} & $P$-value \\
\hline Trunk orientation (deg) & 2902 & 0.809 & $<0.001$ \\
Hip flexion (deg) & 2066 & 0.625 & $<0.001$ \\
Knee flexion (deg) & -1069 & -0.284 & $<0.001$ \\
Ankle dorsiflexion (deg) & 257 & 0.043 & 0.011 \\
Hip flexion diff (deg) & 1280 & 0.564 & $<0.001$ \\
Knee flexion diff (deg) & -428 & -0.169 & $<0.001$ \\
Ankle dorsiflexion diff (deg) & 36 & -0.008 & 0.628 \\
\hline
\end{tabular}

Table 2. Bivariate correlations $r$ between the peak ACL force and each of the predictor variables

\begin{tabular}{lrc}
\hline Variable & $r$ & $P$-value \\
\hline Trunk orientation & 0.787 & $<0.001$ \\
Hip flexion & 0.166 & $<0.001$ \\
Knee flexion & -0.275 & $<0.001$ \\
Ankle dorsiflexion & 0.260 & $<0.001$ \\
Hip flexion diff & 0.138 & $<0.001$ \\
Knee flexion diff & 0.181 & $<0.001$ \\
Ankle dorsiflexion diff & -0.274 & $<0.001$ \\
\hline
\end{tabular}

Table 3. Semipartial correlations $s r$ for the predictor variables

\begin{tabular}{lr}
\hline Variable & \multicolumn{1}{c}{$s r$} \\
\hline Trunk orientation & 0.777 \\
Hip flexion & 0.426 \\
Knee flexion & -0.199 \\
Ankle dorsiflexion & 0.029 \\
Hip flexion diff & 0.388 \\
Knee flexion diff & -0.117 \\
Ankle dorsiflexion diff & 0.006 \\
\hline
\end{tabular}

A biped musculo-skeletal model of a skier was developed and a baseline simulation was successfully obtained in which muscle stimulation patterns of the modeled skier were optimized to track measurement data of a reference landing movement in competitive downhill skiing. During the baseline simulation, the peak tensile force in the ACL amounted to $695 \mathrm{~N}$ (0.9 body weight (BW)) and occurred about $30 \mathrm{~ms}$ after initial ground contact. There is a lack of studies reporting ligament forces during a well-performed jump landing in downhill skiing. In the simulation study of Gerritsen et al. (1996), peak ACL force was reported only for a perturbed off-balance situation and amounted to $1350 \mathrm{~N}$; for the unperturbed simulation, only the net knee reaction force was reported. However, durations of 20-40 ms and magnitudes of up to $0.4 \mathrm{BW}$ were reported in 
previous studies analyzing drop jumps from a $60 \mathrm{~cm}$ platform (Pflum et al., 2004; Kernozek \& Ragan, 2008). The considerably higher peak ACL force in the present study might be primarily due to the effective fall height of about $90 \mathrm{~cm}$. The higher fall height is likely to cause an increased tibiofemoral compression force, which would directly elevate anterior tibial load and consequently ACL load due to the tibial plateau angle (Yeow et al., 2010). Additionally, the low friction between skis and snow reduces the posterior ground reaction force, which is considered to protect the ACL.

The simulation with random perturbations of the orientation of the trunk and the joint angles of the skier prior to ground contact resulted in substantially higher peak ACL forces compared with the baseline simulation. Peak ACL forces reached values up to 2017 N. As the ACL typically tears at loads greater than $2000 \mathrm{~N}$ (Woo et al., 1991), this peak ACL force might have resulted in a possible injury situation. In the simulation study of Kietlinski \& Rzymkowski (2005), also, a high injury risk was reported during a backward off-balance jump landing movement. A peak elongation of the ACL of $20 \%$ was predicted and based on the Abbreviated Injury Scale, a high risk of total rupture was concluded. Based on the study of Kietlinski \& Rzymkowski (2005) and the results of the present simulations, a sagittal plane ACL injury mechanism might exist during jump landing in downhill skiing. Although the current opinion states that it is highly probable that ACL injuries occur during multi-planar mechanisms of injury (Quatman et al., 2010), a single-planar mechanism might be sufficient to tear the ACL during jump landing in downhill skiing.

Multiple regression analysis revealed that the increase of backward lean, hip flexion, knee extension, and ankle dorsiflexion were related to higher peak ACL forces during the subsequent landing movement. These results are in agreement with video sequences of injury cases during landing movements in world cup downhill skiing. Skiers' mistakes such as a poor jumping technique and incorrect tactical decisions have been determined as key factors contributing to injury and these mistakes have been observed to result in a backward off-balance situation of the skier during the flight and in landing on the tails of the skis with nearly extended knees (Bere et al., 2011a). Also, in the computer simulation study of Gerritsen et al. (1996), a perturbed backward off-balance landing movement of an alpine skier resulted in higher ligament forces compared with an unperturbed landing simulation. In other sports, more backward lean and an increased distance between the center of mass and the base of support were identified in ACL injuries cases by videotape analysis (Sheehan et al., 2012). Interestingly, our simulation results are consistent with the results of Sheehan et al. (2012), as backward lean in combination with an increase of hip flexion and knee extension results in an increased distance between the center of mass and the base of support.
The orientation of the trunk prior to landing was identified as the most important predictor for high ACL forces among the perturbed variables. This result reveals a set of preventive measures, which could be easily implemented in training and competitions. To keep the trunk in a forward oriented position during jumping skiers have to rotate their body forward during takeoff and stay in a tuck position during flight. Low speed and a gentle change of the radius of the takeoff area ease the necessary forward rotation of the skier to achieve the required angular momentum. Additionally, a straight and sufficiently long inrun favors a proper preparation of the skiers before takeoff. High flight trajectories are likely to result in a more upright orientation of the trunk during flight and landing. Resulting air resistance may act above the center of mass rotating the skier backward especially when the skier's speed is high. So low flight trajectories and low speed have to be applied in jump design. The inclusion of exercise regimes in ACL injury prevention to improve body position during landing was proposed by Shimokochi et al. (2013), who studied the influence of changing the sagittal plane body position (selfselected, leaning forward and upright) during single-leg landings. Based on the present results, special focus on trunk control regarding jump landing in downhill skiing is suggested.

The musculo-skeletal model had certain limitations. The model was two-dimensional and effects due to internal/external rotation and varus/valgus of the knee joint were not included, which are known to affect ligament forces (Shimokochi \& Shultz, 2008). Such out-ofplane loads are likely to occur during jump landings (Bere et al., 2011a), and our findings do not directly apply to such cases. Extending the present planar model to three dimensions to investigate these out-of-plane loads additionally is in progress.

In each perturbed landing simulation, muscle stimulation patterns were re-optimized such that the skier tries an active recovery motion to regain balance. Because the optimized muscle stimulation patterns affect the corresponding muscle forces and the loading of the knee and knee ligaments, a sensitivity analysis was conducted to test whether the optimized muscle stimulation patterns are affected by the choice of the objective function. The analysis showed that changing the weight factor $w_{\text {mus }}$ by a factor of 2 (setting $w_{\text {mus }}$ to 0.5 and 2, respectively) in a sample of 100 perturbed landing simulations only changed the force in the ACL on average by $4 \%$. Regaining a balanced position represents the behavior that would be expected from a real skier who finds him/ herself in a perturbed position prior to ground contact. Up to the authors' knowledge, this is the first study, where a series of perturbed landing simulations with a given task has been solved. The conventional approach has been to generate repeated forward simulations with the same muscle excitation patterns (McLean et al., 2004,2008 ) or to use a prescribed muscle excitation 
pattern (Gerritsen et al., 1996). However, both approaches neglect task-oriented adaptive behavior of the athlete.

In the series of Monte Carlo simulations, the joint angles and trunk orientation were perturbed prior to ground contact, and not the velocities. Variation of the linear velocity of the trunk segment normal to the snow surface could be used to include the influence of jump height. However, measurement data regarding variation of jump heights in downhill skiing are currently lacking. Further applications of the present musculo-skeletal model might be to study the effect of the inclination of the landing surface, the speed, and the jump height of the skier as well as modifications of the equipment. In addition, alternative control strategies of the skier might also be investigated aiming at a reduction of the peak force in the ACL during jump landing.

\section{Perspectives}

Competitive skiers face a high injury risk during offbalance jump landing maneuvers in downhill skiing (Flørenes et al., 2009, 2012). In vivo studies, which mimic injury situations, are ethically not feasible. However, musculo-skeletal modeling offers the possibility to study injury situations in a computer environment. A two-dimensional musculo-skeletal skier-skis model was developed and applied to investigate a cause-andeffect relationship between landing position and peak ACL force during jump landing. Backward lean of the skier was identified as the most important predictor for high ACL forces. An increase of hip flexion, knee extension, ankle dorsiflexion, and an asymmetric position were also related to higher peak ACL forces. These results are in agreement with previous simulation results (Gerritsen et al., 1996) and video sequences of injury cases during landing movements in competitive downhill skiing (Bere et al., 2011a). The inclusion of exercise regimens in ACL injury prevention programs with special focus on trunk control and a set of preventive measures were suggested. Further applications of the present model might be to study the effect of jump height and speed, modifications of the equipment, and neuromuscular control strategies aiming at the prediction of ACL risk factors during jump landing in downhill skiing.

Key words: skiing, simulation, musculo-skeletal modeling, multiple regression.

\section{Supporting Information}

Additional Supporting Information may be found in the online version of this article at the publisher's web-site:

Video S1. Animation of the baseline landing simulation.

\section{References}

Barelle C, Ruby A, Tavernier M.

Experimental model of the aerodynamic drag coefficient in alpine skiing. J Appl Biomech 2004: 20: 167-176.

Barone M, Senner V, Schaff P. ACL injury mechanism in alpine skiing: analysis of an accidental ACL rupture. In: Johnson RJ, ed. Skiing trauma and safety, 12th vol, ASTM STP 1345. West Conshohocken, PA: ASTM, 1999: 63-81.

Bere T, Flørenes TW, Krosshaug T, Koga $\mathrm{H}$, Nordsletten L, Irving C, Muller E, Reid RC, Senner V, Bahr R. Mechanisms of anterior cruciate ligament injury in World Cup alpine skiing: a systematic video analysis of 20 cases. Am J Sports Med 2011a: 39: 1421-1429.

Bere T, Flørenes TW, Krosshaug T, Nordsletten L, Bahr R. Events leading to anterior cruciate ligament injury in World Cup Alpine Skiing: a systematic video analysis of 20 cases. Br J Sports Med 2011b: 45: 1294-1302.

Betts JT. Practical methods for optimal control and estimation using nonlinear programming. Philadelphia, PA: Society for Industrial and Applied Mathematics, 2010.
Bruck F, Lugner P, Schretter H. A dynamic model for the performance of carving skis. In: Johnson R, Lamont M, Shealy J, eds. Skiing trauma and safety, 14th vol, ASTM STP 1440. West Conshohocken, PA: ASTM, 2003: 10-23.

Flørenes TW, Bere T, Nordsletten L, Heir S, Bahr R. Injuries among male and female World Cup alpine skiers. Br J Sports Med 2009: 43: 973-978.

Flørenes TW, Nordsletten L, Heir S, Bahr R. Injuries among World Cup ski and snowboard athletes. Scand J Med Sci Sports 2012: 22: 58-66.

Gerritsen KG, Nachbauer W, van den Bogert AJ. Computer simulation of landing movement in downhill skiing: anterior cruciate ligament injuries. J Biomech 1996: 29: 845-854.

He J, Levine WS, Loeb GE. Feedback gains for correcting small perturbations to standing posture. IEEE Trans Autom Control 1991: 36: 322-332.

Herzog W, Read LJ. Lines of action and moment arms of the major force-carrying structures crossing the human knee joint. J Anat 1993: 182: 213-230.
Kaps P, Nachbauer W, Mössner M. Determination of kinetic friction and drag area in alpine skiing. In: Mote C, Johnson R, Hauser W, Schaff P, eds. Skiing trauma and safety, 10th vol, ASTM STP 1266. Philadelphia, PA: ASTM, 1996: 165-177.

Kernozek TW, Ragan RJ. Estimation of anterior cruciate ligament tension from inverse dynamics data and electromyography in females during drop landing. Clin Biomech 2008: 23 : 1279-1286.

Kietlinski K, Rzymkowski C. An attempt to predict a risk of some of the knee region ski injuries by means of computer simulations. J ASTM Int 2005: 2: 67-73.

Krosshaug T, Andersen TE, Olsen OE, Myklebust G, Bahr R. Research approaches to describe the mechanisms of injuries in sport: limitations and possibilities. Br J Sports Med 2005: 39: 330-339.

Matsuda S, Miura H, Nagamine R, Urabe K, Ikenoue T, Okazaki K, Iwamoto Y. Posterior tibial slope in the normal and varus knee. Am J Knee Surg 1999: 12: 165-168.

McLean SG, Huang X, Su A, van den Bogert AJ. Sagittal plane biomechanics 
cannot injure the ACL during sidestep cutting. Clin Biomech 2004: 19: 828-838.

McLean SG, Huang X, van den Bogert AJ. Investigating isolated neuromuscular control contributions to non-contact anterior cruciate ligament injury risk via computer simulation methods. Clin Biomech 2008: 23: 926-936.

McLean SG, Su A, van den Bogert AJ. Development and validation of a 3-D model to predict knee joint loading during dynamic movement. J Biomech Eng 2003: 125: 864-874.

Nachbauer W, Kaps P, Nigg BM, Brunner F, Lutz A, Obkircher G, Mössner M. A video technique for obtaining 3-D coordinates in alpine skiing. J Appl Biomech 1996: 12: 104-115.

Pflum MA, Shelburne KB, Torry MR, Decker MJ, Pandy MG. Model prediction of anterior cruciate ligament force during drop-landings. Med Sci Sports Exerc 2004: 36: 1949-1958.
Quatman CE, Quatman-Yates CC, Hewett TE. A 'plane' explanation of anterior cruciate ligament injury mechanisms: a systematic review. Sports Med 2010: 40: 729-746.

Sheehan FT, Sipprell WH, Boden BP. Dynamic sagittal plane trunk control during anterior cruciate ligament injury. Am J Sports Med 2012: 40: 1068-1074.

Shimokochi Y, Ambegaonkar JP, Meyer EG, Lee SY, Shultz SJ. Changing sagittal plane body position during single-leg landings influences the risk of non-contact anterior cruciate ligament injury. Knee Surg Sports Traumatol Arthrosc 2013: 21: 888-897.

Shimokochi Y, Shultz SJ. Mechanisms of noncontact anterior cruciate ligament injury. J Athl Train 2008: 43: 396-408.

Spägele T, Kistner A, Gollhofer A. Modelling, simulation and optimisation of a human vertical jump. J Biomech 1999: 32: 521-530.

Trabachnick BG, Fidell LS. Using multivariate statistics. Bosten, MA: Pearson, 2005: 117-194. van den Bogert AJ, Blana D, Heinrich D. Implicit methods for efficient musculoskeletal simulation and optimal control. Proc IUTAM 2011: 2: 297-316.

Wächter A, Biegler LT. On the implementation of a primal-dual interior point filter line search algorithm for large-scale nonlinear programming. Math Program 2006: 106: $25-57$.

Winter DA. Biomechanics and motor control of human movement. New York: Wiley, 2009.

Woo SL, Hollis JM, Adams DJ, Lyon RM, Takai S. Tensile properties of the human femur-anterior cruciate ligament-tibia complex. The effects of specimen age and orientation. Am J Sports Med 1991: 19: 217-225.

Yeow CH, Lee PV, Goh JC. Direct contribution of axial impact compressive load to anterior tibial load during simulated ski landing impact. J Biomech 2010: 43: 242-247. 